\title{
Reaching Sustainability in Healthcare: Strategies for a Healthy Indoor Air Quality in Healing Environments
}

\author{
Marco Gola ${ }^{1(\bowtie)}$, Gaetano Settimo $^{2}$, and Stefano Capolongo ${ }^{1}$ \\ ${ }^{1}$ Politecnico di Milano, 20133 Milan, Italy \\ marco.gola@polimi.it \\ 2 Istituto Superiore di Sanità, 00161 Rome, Italy
}

\begin{abstract}
Indoor Air Quality (IAQ) is one of the main topics in which governments are focusing. In architectures for health, several researches are reporting a growing number of data analysis and research works in order to improve users' health. Although many studies have been conducted related to the biological and physical risks, the chemical risks have been less investigated and only in some specific functional areas of the hospitals.

Starting from some systematic reviews and research works, the paper aims to identify and list the best healthy practices for an adequate IAQ in inpatient wards. In particular, the handbook lists the solutions and strategies related to chemical pollution, starting from design and management, with a focus on (1) localization of hospitals and inpatient rooms, (2) hospital room, (3) microclimatic parameters, (4) ventilation systems, (5) materials and finishing, (6) furniture and equipment, (7) cleaning products and activities, (8) maintenance and (9) management activities, and (10) users and specific training of the workers.

The multidisciplinary approach emphasizes the need for interdisciplinary knowledge and skills aimed to find solutions able to protect users' health status. The design and management decision-making, ranging from the adequate choices of construction site and hospital exposure, finishing materials, cleaning and maintenance activities, etc., which can affect the IAQ must be carried out based on scientific research and data analysis.
\end{abstract}

Keywords: Indoor Air Quality · Hospital settings · Design and management strategies

\section{The Indoor Air Quality}

\subsection{Health Promotion in Confined Spaces}

Although the Indoor Air Quality (IAQ) has been already argued at the end of Eighties by Fanger [1], only recently it becomes a primary strategy to be considered. The topic needs to be focused because of the increasing of exposed population due to lifestyles and the permanence in confined environments (about $90 \%$ of the day).

Even European Community underlines the priority of energy efficiency strategies, in the same time it recommends to reach healthier indoor environments and the 
development of a specific European strategy on IAQ. Currently several EU countries have introduced in their legislations some actions relating to IAQ [2].

Since several years, critical factors due to the exposure to indoor air pollutants have been a matter of concern for EU legislators, and an increasing number of countries has been addressing policies regarding health promotion strategies. EU has often highlighted the importance to investigate IAQ, the relative impacts on health status and recommendations regarding actions and monitoring activities.

Although the current criticisms, in several countries air quality monitoring are carried out in specific professional workplaces in which chemicals are used, but also in some generic indoor spaces $[3,4]$.

\subsection{The Environmental Quality of Healthcare Settings}

The great importance of the issues connected with IAQ in the hospital settings, and all the other similar facilities, accredited or not by the National Health System (NHS), has urged several research and institutional bodies to investigate the topic and find solutions and strategies for healthy environments (especially in relation to the evaluation of the concentration levels in indoor air of the main chemical and biological pollutants) [5].

In such facilities with high managerial, technological and functional complexity, where the several functional units are designed with specific standards and needs (inpatient and outpatient areas, public ones, rehabilitation, etc.), where are carried out assistance and medical activities, diagnostics, training and research activities, etc., they are characterized by the presence of indoor air of chemical and biological components that can affect the health status of users [4-6].

In these healing spaces in which hospital staff interacts for various operational needs, but also users, specific preventive interventions are required, considering that the exposure of key-actors (from the daily users to the hospital staff), whose roles, knowledge and backgrounds, motivations and individual relationships have changed, becoming increasingly an active component to collaborate for improving the quality of the environments, medical services, takes on particular significance and importance, both for the vulnerabilities of the subjects and for the time spent in indoors [7-11].

In the specific case of the activities carried out, it is essential to consider the relationships between the behaviors and activities of the healthcare staff, and all the other users who live and work the healing spaces (outpatients, visitors, external workers for cleaning and maintenance activities, etc.) with their activities and behaviors $[8,12]$. They are also affected by the quality of the building and daily relationships with the correct application of the organizational and management procedures of medical processes.

The use of technological and ventilation systems designed to perform and satisfy the various tasks in the best technical and economic conditions, the furnishings, the level of use, the ordinary and extraordinary cleaning and sanitization activities, maintenance, procedures and organic management of the regular prevention activities implemented and shared within the structures, etc. are all factors that affect significantly on IAQ and on the state of health and satisfaction of users [12, 13].

For this reason, these aspects are increasingly steps of the quality of services, therapies, healthcare services, activities and training program continuously provided, 
contributing to obtaining an effective and adequate quality of the indoor air that responds to the main references elaborated by the World Health Organization (WHO) [14], and which constitute a concrete contribution on a global scale. The WHO activities on air quality have boosted the legislative activities already in place in various European countries that set the maximum concentrations allowed in the indoor air of individual pollutants $[2,14]$.

\section{Risks Correlated to Healing Spaces}

\subsection{Health Promotion in Confined Spaces}

Indoor pollution is defined as "[...] the presence of physical, chemical and biological contaminants in confined spaces' air does not present in outdoor air of high quality ecological systems" [15].

Indoor air pollution is a public health issue. It is related to many of the major chronic diseases, related to respiratory, mucous membranes, skin, nervous and immune systems, such as asthma, fever by humidifiers, allergic alveolitis, legionellosis, etc. In addition to these well-known diseases, several symptoms, very frequently, characterized by neurosensory effects that determine discomfort, decreased wellness of occupants and negative perception of IAQ, may manifest. All the pathologies and diseases are caused by IAQ that affect mainly vulnerable population, in particular elderly, children and those who already suffer for chronic diseases [16].

As WHO highlights, the well-known concepts related to air quality are $[17,18]$ :

- people spend $90 \%$ of their time in confined spaces (working and living places, transports, etc.);

- indoor air is much more polluted than the outdoor, because additional factors related to the indoor space affect air;

- it is estimated that $50 \%$ of diseases are caused by an inadequate IAQ.

In average, an adult inhales and exhales about $8 \mathrm{~L}$ of air per minute, at rest. Therefore, in a day, totally, he breathes about 11 thousand liters of air [19]. Unhealthy air quality can cause several health effects [20].

In general, healing environments must be aimed to users' well-being but, paradoxically, sometimes they become unhealthy. The main factors of air pollution in healthcare facilities are substances used for therapeutic and diagnostic aims, maintenance and cleaning products, disinfectants, ventilation and air conditioning management, heating systems, building materials, furniture and finishing, equipment, etc. [5]. In addition, also the outdoor greatly affects the indoor air, because it is the sum of the outdoor one with the influence of internal factors [4, 12].

Starting from the merging of all these factors, the outcomes cannot be considered acceptable and consequently poor ventilation and inadequate air exchange can cause inadequate conditions. Indoor air in buildings is affected by outdoor and, therefore, by factories in the neighbourhood, road traffic, parking lots, warehouses of irritating substances or waste, construction sites or any other source of air pollution itself [12]. 
Therefore, to guarantee safety and air quality, it should be considered carefully the location of the outer air intakes for ventilation systems and their filters [19, 21].

The presence of contaminants within healthcare environments influences the IAQ compromising wellbeing and safety conditions that must be fundamentals in an adequate hospital complex [22].

Indoor pollutants concentration is inconstant during the time; it is related to the year of construction of the building, its dimensional aspects, the typology of the sources, such as the activities carried out by users, ventilation systems, procedures, cleaning and maintenance activities, etc. [6]. Indoor air, often characterized by a mixture of compounds very variable from outdoor one, can present concentration values of pollutants higher than those external environments and, sometimes, some indoor pollutants are not related to outdoor [4].

Italy lacks of legislations and norms about physical, biological, and chemical pollution of air in functional units. Currently the Legislative Decree No. 81 (2008) is used as a reference document, even if it states guidelines about workplaces [2, 5].

The healthcare facility, as a public infrastructure that hosts many and several users with different health conditions, cultures and origins, is the architecture with the highest number of risk factors and air pollutions [10]. Moreover, to traditional outdoor sources of pollution, occupants and building factors, it is important to add those relating to therapeutic and medical activities and products.

Since sources of chemical, biological and physical pollutants are several and many contaminants and pollutants can influence air quality in healing environments, it is fundamental to guarantee an adequate air-change through proper design of ventilation systems, that must also ensure to filter and enter the best outdoor air, minimizing pollution related to products and activities, materials and diluting the pollutant concentration and drain them [8].

\subsection{Factors that Affect the IAQ in the Environmental Units}

From the chemical pollution's point of view, hospital planners, managers and hospital staff therefore have the strategic role on defining strategies for maximum infection control. In case of design mistakes, it becomes strategic to ensure proper management of the facilities, in particular with the HVAC system with constant air change, choosing furniture and building material with low volatile organic compounds (VOCs) emissions [18], regular and conscious maintenance, medical activities and cleaning procedures (avoiding the use of sprays and other cleaning materials that generate VOCs, etc.) that take place inside the healing spaces [12].

Starting from these considerations, a research group gave rise to a monitoring activity of air quality in inpatient rooms, focusing on chemical pollution for understanding the current values and room features, maintenance activities and medical procedures [23]. For supporting the research project, the authors have done a systematic review on the current State of the Art and knowledge related to chemical pollution in healing spaces and the emerging strategies, supported by scientific data, for healthy inpatient rooms and their indoor air. The focus of the investigation is strictly related to inpatient wards. 


\subsection{How to Assess the Chemical Pollution in Healing Spaces}

Indoor monitoring activities and the assessment of health risk factors are key-points for identifying the measures needed to prevent and/or to reduce pollutants' concentrations.

To program the monitoring activity and identify the appropriate sampling techniques, it is necessary to define the compounds that must be taken into account $[5,10]$.

Sampling methods vary, depending on the type of pollutant to be analysed and they can be subdivided in active, passive and canister samplings. These can be defined by a preliminary assessment through screening that allows to analyse the emission pattern of the sources, providing information for defining the type of sampling and analysis.

For a first analysis of air concentrations, particular attention is necessary for indoor pollutant classes of VOCs and Particulate Matter (PM).

WHO proposes a classification of VOCs in 4 groups (very volatile, volatile, semivolatile, associated with particulate matter) based on boiling limits. These classes belong to species that have been identified as hazardous to human health, including pollutant by the International Agency for Research on Cancer 1 as certain carcinogens.

Determination of the main indoor pollutants can be done with passive methods, which involves sampling and subsequent laboratory analysis of collected samples, or active ones, in situ, with direct-fit systems. In addition, in relation to the duration of sampling, monitoring is defined as short-term (from a few seconds to a few hours) or long-term (generally over $8 \mathrm{~h}$ ).

For each of them, it is in any case to use and adopt the correct guidelines by EN ISO 16000, because only with the use of efficient instrumentations and an adequate protocol the outcomes are trusted. Nowadays, the market offers several products that are not enough sensitive and with unreliable results (Table 1).

Table 1. Indoor air factors in inpatient room [6].

\begin{tabular}{l|l|l|l}
\hline Criterion & Field of interest & Influence & Focus \\
\hline Design factors & $\begin{array}{l}\text { They refer to all the } \\
\text { components that } \\
\text { characterize the } \\
\text { inpatient room (room } \\
\text { dimensions, furniture, } \\
\text { finishing, etc.) }\end{array}$ & $\begin{array}{l}\text { Their emissions are } \\
\text { constant, although in } \\
\text { relation to their life } \\
\text { the emissions may } \\
\text { decrease over the } \\
\text { time }\end{array}$ & $\begin{array}{l}\text { dimensional aspects, } \\
\text { room configuration } \\
\text { and door motion } \\
\text { finishing materials and } \\
\text { furniture }\end{array}$ \\
\hline $\begin{array}{l}\text { Management } \\
\text { and cleaning } \\
\text { activities }\end{array}$ & $\begin{array}{l}\text { They refer to the } \\
\text { management and } \\
\text { maintenance } \\
\text { activities, ventilation } \\
\text { systems, cleaning and } \\
\text { disinfectant activities, } \\
\text { etc. carried out in the } \\
\text { room and in the } \\
\text { functional units }\end{array}$ & $\begin{array}{l}\text { They can highly } \\
\text { affect the indoor air, } \\
\text { but their emissions } \\
\text { can be controlled } \\
\text { through the } \\
\text { Applications of } \\
\text { strategies, and in the } \\
\text { same time, they can } \\
\text { be changes if their } \\
\text { actions are dangerous } \\
\text { for users }\end{array}$ & $\begin{array}{l}\text { cleaning and } \\
\text { maintenance activities } \\
\text { ventilation systems } \\
\text { maintenance and } \\
\text { operational strategies }\end{array}$ \\
& &
\end{tabular}


Table 1. (continued)

\begin{tabular}{l|l|l|l}
\hline Criterion & Field of interest & Influence & Focus \\
\hline $\begin{array}{l}\text { Human } \\
\text { presence and }\end{array}$ & $\begin{array}{l}\text { They refer to the } \\
\text { presence of users, } \\
\text { their health status, } \\
\text { and the medical } \\
\text { activities carried out } \\
\text { in the inpatient room }\end{array}$ & $\begin{array}{l}\text { Their presence and } \\
\text { application can vary, } \\
\text { and therefore they } \\
\text { can affect the indoor } \\
\text { air in different modes. } \\
\text { In general this } \\
\text { component does not } \\
\text { affect highly the } \\
\text { indoor air } \\
\text { performances }\end{array}$ & $\begin{array}{l}\bullet \text { human behavior } \\
\text { medical activities }\end{array}$ \\
\hline $\begin{array}{l}\text { Outdoor and } \\
\text { microclimatic } \\
\text { factors }\end{array}$ & $\begin{array}{l}\text { They refer to the } \\
\text { outdoor air, the solar } \\
\text { exposure and } \\
\text { microclimatic } \\
\text { parameters }\end{array}$ & $\begin{array}{l}\text { Although these } \\
\text { factors can vary, they } \\
\text { have a great influence } \\
\text { on the indoor air and } \\
\text { the performances of } \\
\text { materials in the room } \\
\text { and air fluxes }\end{array}$ & $\begin{array}{l}\text { - outdoor issues and } \\
\text { site localization } \\
\text { microclimatic } \\
\text { parameters } \\
\text { solar exposure }\end{array}$ \\
\hline
\end{tabular}

\section{The Handbook of Best Practices for Inpatient Room}

\subsection{Localization of the Hospital and Inpatient Room}

The localization of the healthcare facility is strategic for the relationships between outdoor and indoor. It is clever that the concentration of pollutants in outdoor air is lower than in indoor ones. For this reason, for reducing the outdoor concentrations of chemical pollutants in hospital environments, the site selection is of primary importance [12].

In addition, air filtration and air intakes of ventilation systems, if adequately localized, can reduce the input of contaminants coming from outdoor into the hospital settings.

Typically, the inpatients areas are in the upper floors. They can be a double, triple or five-fold body, in which the inpatient rooms are localized on the external sides [19, 21]. The building typology and the organization of the project area can influence the solar exposures of the room and it can highly affect indoor air. As the scientific literature and several case studies demonstrated, in relation to the pollution in the neighbourhood and solar exposure, strategies in ventilation system, with high performances and filtering systems, and technological strategies for reducing solar intake can be applied [19].

\subsection{Design of the Hospital Room}

Inpatient room requires several attentions because they should respond to the patients, medical staff and visitors' needs. Well-designed rooms can speed up healing and, in 
addition, decrease the rate of errors in the process [22,24]. As the scientific literature and several simulations evidenced, there is not any specific dimension of the double and single rooms. It is strongly affected by several variable defined by ventilation systems, human density, window openings, etc. and the layouts' design of the project [6].

For a good IAQ in rooms, it is necessary to pre-assess the possible factors that influence the performances and impacts related to selected furniture, finishing materials, microclimatic parameters, ventilation system, room's volume, and medical activities. The door and window openings can highly affect the performances of the healing unit [12].

\subsection{Microclimatic Parameters}

Microclimatic parameters $\left(\mathrm{CO}_{2}\right.$, temperature, relative humidity and air velocity) have a great influence in indoor air. As the scientific literature evidenced, the increase of microclimatic parameters can affect the performances of workers and patients, finishing materials and furniture, as well as the chemical emissions of cleaning products.

An adequate microclimatic parameters, supported by the window opening and efficient ventilation system, can guarantee good performances in IAQ and users' comfort [1].

\subsection{Ventilation Systems}

In inpatient areas, the influence of medical activities, high temperature for patient wellbeing, users' health status and behavior, etc. can affect IAQ performances. It is necessary an adequate ventilation system to control indoor pollutants through their removal by dilution, air purification and filtration [21]. As the scientific literature evidenced, insufficient ventilation and filtration contribute to poor IAQ for outdoor contaminants in the building, recirculation of indoor pollutants and dirt build-up in air handling systems [19, 21]. In general, ventilation systems should be designed specifically for the activities to be carried out, managed and well-filtered for guaranteeing the better performances during its functioning [19].

\subsection{Construction and Finishing Materials}

In order to minimize the sources of pollution, it is necessary to identify and define adequate materials that respond to the medical and hygiene requirements. Hospital planners should also consider that environmental conditions of the space can affect the release of pollutants, such as high-temperature or humidity that can increase material emissions, solar exposure, etc. [6, 25].

For achieving healthier environments, as some researchers have highlighted, it is necessary an adequate materials' selection with low VOC emissions, as less porous as possible for supporting the performances of cleaning activities and easy maintenance [25].

In general, finishing and furniture must comply with international certifications, and during and after their installation, they need of an adequate ventilation for diluting their emissions, for several days (at least $72 \mathrm{~h}$ ) [12]. 


\subsection{Furniture and Equipment}

In order to reduce the sources of pollution, it is important to select furniture and equipment that respond to hygiene requirements and medical activities to be carried out. As the scientific literature evidenced, it is necessary an adequate selection of furniture with low or zero VOC emissions, as less porous as possible to facilitate easy maintenance and cleaning procedures. Furniture should be placed in indoor environments after the site construction have been completed [12]. Furniture must comply with international certifications, and during and after their installation, they need of an adequate ventilation for diluting their emissions, for several days (at least $72 \mathrm{~h}$ ) [25]. In order to avoid the emission of contaminants due to deterioration, finishing materials and furnishings must be periodically checked and maintained for ensuring that equipment and furnishings are placed in positions where they do not interfere with airflows [12].

\subsection{Cleaning Products and Procedures}

A clean space is an essential requirement for an adequate IAQ. However, inadequate detergents and/or cleaning procedures may cause adverse effects on air performances. Proper ventilation must be ensured during and immediately after cleaning procedures. Several studies suggest window opening for 10-15 min, in the absence of users [5].

Cleaning activity should be defined through specific protocols, and medical and non-medical staff should be trained on risks. It is recommended the selection of minimum emission cleaning products, adequate for the surfaces to be treated $[6,12]$.

\subsection{Maintenance Activities}

It is fundamental that hospitals, as well as well-designed and built to ensure adequate IAQ, must be regularly maintained in all its components, for high performances [6, 26].

As several researchers highlight, a regular and constant maintenance and management program can facilitate the coordination of the necessary activities to be carried out.

Therefore periodic and regular inspections and investigations in hospital settings and checks of the quality and efficiency, identification of potential air pollution by maintenance activities, analysis of datasheet of cleaning products, construction materials, furnishings, and medical equipment with low VOC emissions should be verified [12].

\subsection{Management Activities}

It is fundamental that hospitals, as well as well-designed and built to ensure adequate IAQ, must be well managed, for ensuring all the accurate work of hospital planners would not be productive, and to be health promoter [8]. An adequate IAQ management program can facilitate the coordination of the necessary activities to identify, correct, and prevent IAQ issues and to overcome critical factors with design and management strategies [8, 12]. Regular monitoring activities permits to the healthcare organization to know the performances of the healthcare facility. In addition, air samplings and the 
warranty of adequate values can guarantee to the hospital managers, in case of discomfort and a complaint from workers and patients, to have data that certify the correct provision of medical activities and the quality of healing environments [27]. An IAQ manager can guarantee a regular control and efficiency of the hospital [12].

\subsection{Users and Workers' Behaviour}

Every day, several users pass through the inpatient ward: from hospital staff (medical one, cleaners and maintainers) to patients and their relatives. Although the scientific literature evidenced that users' presence in the inpatient room does not highly influence chemical emission in comparison with other factors, but their actions and behaviours, as well as their behaviour and health status, can affect IAQ performances [6]. It is requested an adequate behaviour by all the users for guaranteeing the best performances of the room, without (in any way) affecting the health status of patients [12].

\section{Conclusions}

IAQ is a very broad topic in which any variable can affect the performances in indoor environments. Design and management strategies which may be adequate, in relation to different procedures, can decrease or increase the quality of performances.

The considerations emerged from the research are aimed at project managers, designers, technicians and all those who can contribute to the reduction of health risks.

It is clear that the decision-making in design and management issues ranging from the adequate choices of construction site and hospital exposure, finishing materials and furniture, cleaning and maintenance activities, etc., that can affect the IAQ, must be carried out on the basis of scientific researches and data [28, 29]. It is further necessary that the decision-maker should be composed by different professionals in order to guarantee a multidisciplinary and synergic design project in its various aspects.

Hospitals are complex, with different needs, users and requirement compared to other building facilities, and they work $24 / 7$. For this reason, every action should be assessed in relation to their performances and to interrupt medical activities as little as possible.

The tools can be used autonomously and/or synergistically for supporting the decision making of chief medical directors and managers of the facilities, and the implementation of strategies during the time for permitting the best performances of the healing environments (i.e. adopting design strategies and verifying subsequently the room performances, verifying the procedures and monitoring the benefits of indoor air, etc.).

\section{References}

1. Fanger, P.O., Melikov, A.K., Hanzawa, H., Ring, J.: Air turbulence and sensation of draught. Energies Build. 12, 21-39 (1988). https://doi.org/10.1016/0378-7788(88)90053-9 
2. Settimo, G.: Existing guidelines for indoor air quality: the case study of hospital environments. In: Capolongo, S., Settimo, G., Gola, M. (eds.) Indoor Air Quality in Healthcare Facilities. SPH, pp. 13-26. Springer, Cham (2017). https://doi.org/10.1007/9783-319-49160-8_2

3. D'Amico, A., Fara, G.M.: The need to develop a multidisciplinary expertise for the microbiological safety of operating theatres. Ann. Ig. 28(6), 379-380 (2016). https://doi.org/ 10.7416/ai.2016.2119

4. Gola, M., Settimo, G., Capolongo, S.: Indoor air in healing environments: Monitoring chemical pollution in inpatient rooms. Facilities 37(9/10), 600-623 (2019). https://doi.org/ 10.1108/F-01-2018-0008

5. Settimo, G., Bonadonna, L., Gherardi, M., di Gregorio, F., Cecinato, A., et al. (eds.): Qualità dell'aria negli ambienti sanitari: strategie di monitoraggio degli inquinanti chimici e biologici. Rapporti ISTISAN 19/17. Istituto Superiore di Sanità, Rome (2019). Istituto Superiore di Sanità. http://old.iss.it/binary/publ/cont/19_17_web.pdf. Accessed 29 Jan 2020

6. Gola, M., Settimo, G., Capolongo, S.: Indoor air quality in inpatient environments: a systematic review on factors that influence chemical pollution in inpatient wards. J. Healthc. Eng. 2019, 8358306 (2019). https://doi.org/10.1155/2019/8358306

7. Smith, D., Alverdy, J., An, G., Coleman, M., Garcia-Houchins, S., Green, J., et al.: The hospital microbiome project: meeting report for the 1st hospital microbiome project workshop on sampling design and building science measurements. Stan. Genomic Sci. 8, 112-117 (2013). https://doi.org/10.4056/sigs.3717348

8. Leung, M., Chan, A.H.S.: Control and management of hospital indoor air quality. Med. Sci. Monit. 12, SR17-SR23 (2006)

9. Harvey Jr., T.E., Pati, D.: Keeping watch. Design features to aid patient and staff visibility. Health Facil. Manag. 25(6), 27-31 (2012)

10. Śmiełowska, M., Marć, M., Zabiegała, B.: Indoor air quality in public utility environmentsa review. Environ. Sci. Pollut. Res. 24(12), 11166-11176 (2017). https://doi.org/10.1007/ s11356-017-8567-7

11. ISIAQ (International Society of Indoor Air Quality and Climate): Review on indoor air quality in hospitals and other health care facilities, vol. 43. International Society of Indoor Air Quality and Climate, Philadelphia (2003)

12. Gola, M., Settimo, G., Capolongo, S.: Chemical pollution in healing spaces: the decalogue of the best practices for adequate indoor air quality in inpatient rooms. Int. J. Environ. Res. Public Health 16(22), 4388 (2017). https://doi.org/10.3390/ijerph16224388

13. de Oliveira, A.C., Damasceno, Q.S.: Surfaces of the hospital environment as possible deposits of resistant bacteria: a review. Rev. Esc. Enferm. USP 44(4), 1112-1117 (2010). https://doi.org/10.1590/S0080-62342010000400038

14. Bonadonna, L., de Grazia, M.C., Capolongo, S., Casini, B., Cristina, M.L., et al.: Water safety in healthcare facilities. The Vieste Charter. Ann. Ig. 29(2), 92-100 (2017). https://doi. org/10.7416/ai.2017.2136

15. Ministero dell'Ambiente: Relazione della Commissione nazionale per l'inquinamento degli ambienti confinati. Ministero dell'Ambiente, Rome (1991)

16. Gianfredi, V., Grisci, C., Nucci, D., Parisi, V., Moretti, M.: Communication in health. Recenti Prog. Med. 109(7-8), 374-383 (2018). https://doi.org/10.1701/2955.29706

17. D’Alessandro, D., Tedesco, P., Rebecchi, A., Capolongo, S.: Water use and water saving in Italian hospitals. A preliminary investigation. Ann Ist Super Sanità 52(1), 56-62 (2016). https://doi.org/10.4415/ANN_16_01_11

18. WHO: Guidelines for Indoor Air Quality: Selected Pollutants. WHO, Copenhagen (2010) 
19. Moscato, U., Borghini, A., Teleman, A.A.: HVAC management in health facilities. In: Capolongo, S., Settimo, G., Gola, M. (eds.) Indoor Air Quality in Healthcare Facilities. SPH, pp. 95-106. Springer, Cham (2017). https://doi.org/10.1007/978-3-319-49160-8_9

20. Campanella, P., Azzolini, E., Izzi, A., Pelone, F., De Meo, C., La Milia, D., Specchia, M., Ricciardi, W.: Hospital efficiency: How to spend less maintaining quality? Annali dell'Istituto Superiore di Sanita 53(1), 46-53 (2017). https://doi.org/10.4415/ANN_17_ 01_10

21. Joppolo, C.M., Romano, F.: HVAC system design in healthcare facilities and control of aerosol contaminants: issues, tools, and experiments. In: Capolongo, S., Settimo, G., Gola, M. (eds.) Indoor Air Quality in Healthcare Facilities. SPH, pp. 83-94. Springer, Cham (2017). https://doi.org/10.1007/978-3-319-49160-8_8

22. Brambilla, A., Capolongo, S.: Healthy and sustainable hospital evaluation-A review of POE tools for hospital assessment in an evidence-based design framework. Buildings 9(4), 76 (2019). https://doi.org/10.3390/buildings9040076

23. Settimo, G., Gola, M., Mannoni, V., De Felice, M., Padula, G., Mele, A., Tolino, B., Capolongo, S.: Assessment of indoor air quality in inpatient wards. In: Capolongo, S., Settimo, G., Gola, M. (eds.) Indoor Air Quality in Healthcare Facilities. SPH, pp. 107-118. Springer, Cham (2017). https://doi.org/10.1007/978-3-319-49160-8_10

24. Brambilla, A., Buffoli, M., Capolongo, S.: Measuring hospital qualities. A preliminary investigation on Health Impact Assessment possibilities for evaluating complex buildings. Acta bio-medica: Atenei Parmensis 90(9S), 54-63 (2019). https://doi.org/10.23750/abm. v90i9-S.8713

25. Gray, W.A., Vittori, G., Guenther, R., Vernon, W., Dilwali, K.: Leading the way: innovative sustainable design guidelines for operating healthy healthcare buildings. In: Proceedings of the ISIAQ-10th International Conference on Healthy Buildings, Curran Associates, Red Hook, pp. 1212-1217 (2012)

26. Pati, D., Park, C.-S., Augenbroe, G.: Facility maintenance performance perspective to target strategic organizational objectives. J. Perform. Constr. Facil. 24(2), 180-187 (2010). https:// doi.org/10.1061/(ASCE)CF.1943-5509.0000070

27. Gola, M., Mele, A., Tolino, B., Capolongo, S.: Applications of IAQ monitoring in international healthcare systems. In: Capolongo, S., Settimo, G., Gola, M. (eds.) Indoor Air Quality in Healthcare Facilities. SPH, pp. 27-39. Springer, Cham (2017). https://doi.org/10. 1007/978-3-319-49160-8_3

28. Gola, M., Signorelli, C., Buffoli, M., Rebecchi, A., Capolongo, S.: Local health rules and building regulations: a survey on local hygiene and building regulations in italian municiples. Ann. Istituto Superiore di Sanità 53(3), 223-230 (2017). https://doi.org/10.4415/ ANN_17_03_08

29. Sotgiu, G., Aliberti, S., Piana, A.: Nosocomial transmission of carbapenem-resistant Klebsiella pneumoniae in an Italian university hospital: a molecular epidemiological study. J. Hosp. Infect. 99(4), 413-418 (2018). https://doi.org/10.1016/j.jhin.2018.03.033 
Open Access This chapter is licensed under the terms of the Creative Commons Attribution 4.0 International License (http://creativecommons.org/licenses/by/4.0/), which permits use, sharing, adaptation, distribution and reproduction in any medium or format, as long as you give appropriate credit to the original author(s) and the source, provide a link to the Creative Commons license and indicate if changes were made.

The images or other third party material in this chapter are included in the chapter's Creative Commons license, unless indicated otherwise in a credit line to the material. If material is not included in the chapter's Creative Commons license and your intended use is not permitted by statutory regulation or exceeds the permitted use, you will need to obtain permission directly from the copyright holder.

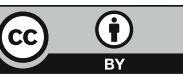

